\title{
Symptomatic Narcolepsy in Sjögren Syndrome
}

EUN-KEE BAE, MD, PhD, Department of Neurology, Inha University Hospital, Incheon, Korea; DAN A. OH, MD, Department of Neurology, Inha University Hospital, Incheon, Korea; BYUNG-NAM YOON, MD, Inha University Hospital, Neurology, Incheon, Korea. Supported by an Inha University research grant (INHA-50479). Address correspondence to Dr. E.K. Bae, Department of Neurology, Inha University Hospital, 7-206, 3-Ga, Sinheung-Dong, Jung-Gu, Incheon, 400-711, Republic of Korea. E-mail: alchemist0210@ gmail.com. J Rheumatol 2017;44:852-3; doi:10.3899/jrheum.160940.

Department/institution policy currently does not require approval of the ethics board for case reports.

Narcolepsy is a debilitating sleep disorder typically characterized by excessive daytime sleepiness with or without cataplexy. The symptoms of narcolepsy can occur during the course of other neurological conditions (i.e., symptomatic narcolepsy), such as inherited disorders, tumors, and head trauma 1. Herein, we describe an unusual case of Sjögren syndrome (SS) presented as narcolepsy secondary to bilateral hypothalamic lesions.

A 61-year-old woman presented with excessive daytime sleepiness and fatigue starting a few months previous. She did not have cataplexy, sleep paralysis, or hallucinations. In neurologic examination, her mental status was alert and there were no focal neurologic deficits. Her brain magnetic resonance imaging (MRI) showed T2 high signal intensities in bilateral hypothalamic areas (Figure 1). Cerebrospinal fluid analysis was normal. Polysomnography and multiple sleep latency test revealed shorted sleep latency $(0.2 \mathrm{~min})$ and 2 occurrences of sleep-onset rapid eye movement sleep among 5 nap trials, compatible with the diagnostic criteria of narcolepsy. Serum anti-SSA(Ro) and antiaquaporin-4 antibodies were positive; however, anti-SSB(La) and antinuclear antibodies were negative. She had chronic dry eyes and mouth in a detailed history-taking, and Schirmer test was positive. She was finally diagnosed with SS 2 and neuromyelitis optica spectrum disorder (NMOSD). Although many autoimmune disorders including SS or narcolepsy can coexist with NMOSD 3, to our knowledge polysomnography-proven symptomatic narcolepsy in SS has not been reported to date.
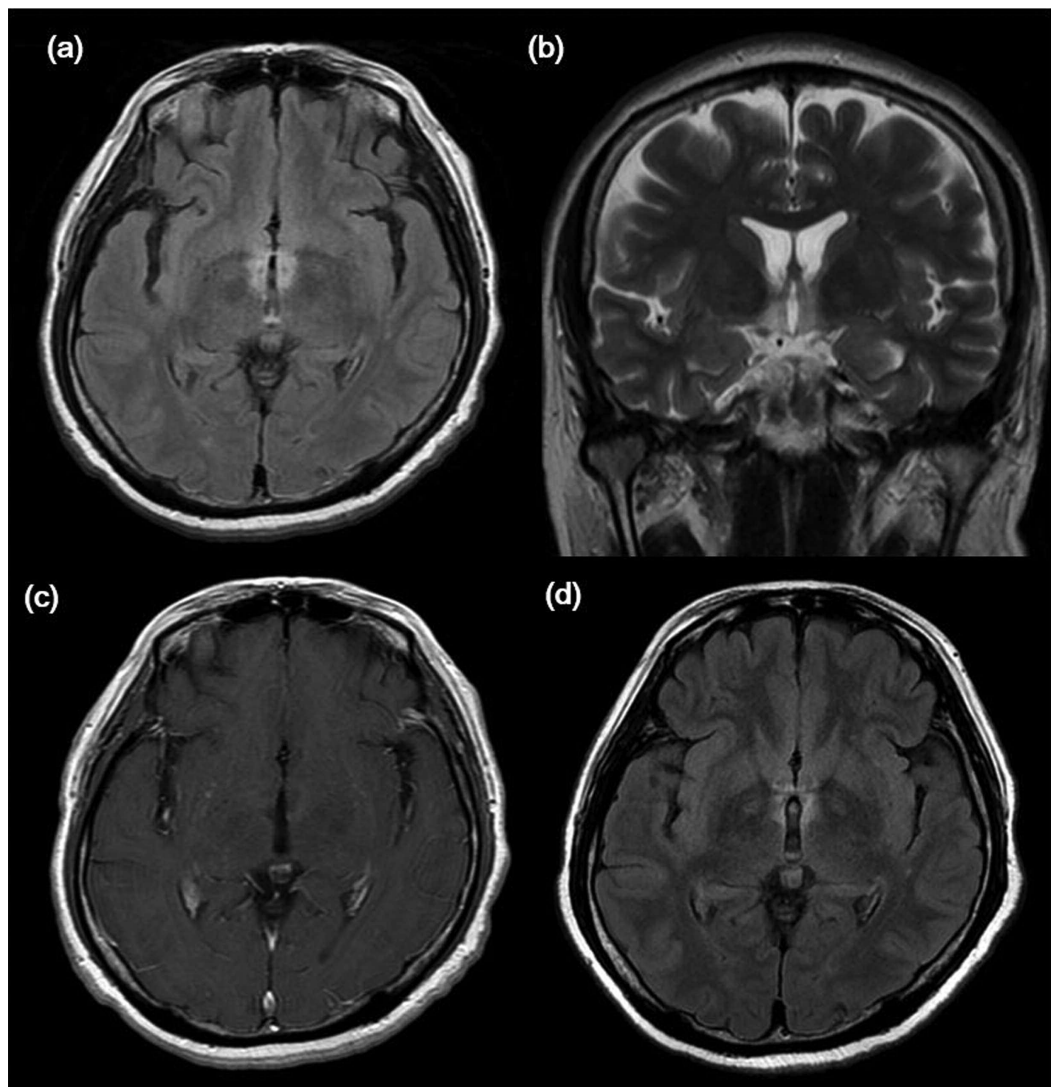

Figure 1. Brain magnetic resonance imaging showed FLAIR (fluid attenuated inversion recovery; a: axial) and T2 (b: coronal) high-signal intensities in bilateral hypothalamic areas. Panel (a) and (b) lesions were not gadolinium-enhanced (c). Although the extent of lesions was slightly decreased, they still remained 3 weeks later $(\mathrm{d})$. 


\section{REFERENCES}

1. Nishino S, Kanbayashi T. Symptomatic narcolepsy, cataplexy and hypersomnia, and their implications in the hypothalamic hypocretin/orexin system. Sleep Med Rev 2005;9:269-310.

2. Vitali C, Bombardieri S, Jonsson R, Moutsopoulos HM, Alexander EL, Carsons SE, et al; European Study Group on Classification Criteria for Sjögren's Syndrome. Classification criteria for Sjogren's syndrome: a revised version of the European criteria proposed by the American-European Consensus Group. Ann Rheum Dis 2002;61:554-8.

3. Iyer A, Elsone L, Appleton R, Jacob A. A review of the current literature and a guide to the early diagnosis of autoimmune disorders associated with neuromyelitis optica. Autoimmunity 2014;47:154-61. 